\title{
UNDERSTANDING CHARACTERISTICS OF THE TRANSFER OF MENTAL MODELS IN THE USE OF ELECTRICAL DEVICES
}

\author{
Kanji TANAKA ${ }^{1)}$ and Toshiki YAMAOKA ${ }^{2)}$ \\ ${ }^{1)}$ Research Center for Advanced Science and Technology, The University of Tokyo, Japan \\ ${ }^{2)}$ Faculty of Systems Engineering, Wakayama University, Japan
}

\begin{abstract}
It has been emphasized that understanding mental models is critical in problem solving, design, and reasoning. It also has been claimed that grasping mental models is difficult because mental models likely depend on our knowledge and experience. We focused attention on the transfer of mental models because we assumed that transfer probably has some common cognitive characteristics. In this study, the transfer group (A-B) and the non-transfer group (B) were prepared. In the transfer group, participants operated product $\mathrm{A}$ and product $\mathrm{B}$ in turn. By contrast, participants in the non-transfer group operated product $\mathrm{B}$ first. Operational time for product $\mathrm{B}$ in both groups was compared to examine how well mental models were transferred. We found that reducing the number of operational procedures could trigger the positive transfer of mental models if the other operational procedures were congruent.
\end{abstract}

Key words: mental models, transfer, operational procedure

Rapid development of information technology has changed our life and society considerably. Many electric products that we use in daily life have complex systems and numerous functions. Since ISO13407 was instituted in 1999, human centeredness has been encouraged. In particular, considering mental models is essential for users to understand system behaviors properly. Kieras and Bovair (1984) showed the role of a mental model in learning how to operate an unfamiliar device. They revealed that having a mental model for the device improved performance on learning and retaining the operating procedures for a device. Several researchers (Halasz \& Moran, 1983; Borgman, 1986) also have reported that having a mental model was one of the most important factors in problem solving. Next, Furuta and Komazaki (1995) examined a process of formation of mental models, analyzing verbal protocols of participants operating a water tank system without prior knowledge of the system. They finally elucidated that participants spontaneously formed several mental models. Numerous works regarding mental models (Johnson-Laird, 1983; de Kleer \& Brown, 1983; Forbus, 1983; Gentner \& Gentner, 1983) have been examined, and the term "mental models" has had many definitions (Doyle \& Ford, 1998). In this study, we define mental models as mental representations to understand behaviors of external systems. Although numerous previous works have investigated mental models, few designs have been produced, which consider mental models, such as product and user interface designs, because these researches

Correspondence concerning this article should be addressed to Kanji Tanaka, Cognitive Science Lab., Research Center for Advanced Science and Technology, The University of Tokyo, 4-6-1, Komaba, Meguroku, Tokyo 153-8904, Japan (e-mail: kanji@fennel.rcast.u-tokyo.ac.jp). 
mainly focused on mental models that have already been completed and these researches did not cover a variety of users. Therefore, we do not have numerous findings about mental models that are beneficial to industry. Baltes (1987) reported that fluid intelligence starts decreasing after the adolescent period and elderly people often use crystallized intelligence; differences in regard to intelligence between older and younger adults were obvious. Moreover, Okawa (1989) reported that individual differences among elderly people regarding intelligence were quite large, and intelligence mainly depended on their background, including occupation and education. Another article disclosed four user characteristics that affected users' mental models of information retrieval systems: educational and professional status, first language, academic background, and computer experience (Zhang \& Chignell, 2001). Thus, it is clear that mental models are composed of numerous factors and have many individual differences. Moreover, Norman (1983) remarked that mental models were incomplete, unstable, unscientific, and parsimonious. Consequently, we face a dilemma in that we have numerous factors to consider in researching mental models, but at the same time, investigating mental models is believed to be necessary for development of industry.

As it seems to be difficult to grasp mental models themselves, we will examine a closely related area of mental models rather than the actual mental models. Kaiho (1988) proposed the ability of transfer in terms of mental models. Transfer of mental models indicates how well people transfer a mental model to another similar situation. A previous study regarding transfer claimed that transfer among different kinds of computer systems, in comparison with simpler machines like tape recorders and calculators, was relatively difficult (Nakatani \& Rohrlich, 1983). Singley and Anderson $(1985,1989)$ conducted a study of lateral transfer - the transfer of mental models at the same level of complexity. In general, the study of transfer is the study of how knowledge acquired in one situation applies (or fails to apply) in other situations. They adapted text editors, UNIX ED, VMS EDT, and UNIX EMACS, as the experimental device. The participant's task was to correct the errors. Singley and Aderson compared the transfer of mental models in terms of performance and keystrokes. As a result, they revealed that positive transfer could bring reduction in total performance time and keystrokes. In particular, reduction in planning time was notable. The study concluded that a very high level of positive transfer was observed between text editors. However, this is likely due to the learning effect and does not remark on conditions that could result in positive transfer. Thus, it is essential to investigate the characteristics of positive transfer of mental models.

The purpose of this study was to examine the characteristics of transfer of mental models, with consideration for differences among individuals. To determine how people transfer their mental models, it was necessary to compare the transfer group with the nontransfer group. Positive transfer describes a situation in which we can easily transfer our familiar mental models to another unfamiliar situation. In the field of product development, product version upgrade is necessary to keep up with the rapid development of information technology. Every time the latest version is upgraded, users must again learn how to use a product. Thus, grasping the characteristics of transfer of mental models will likely make it possible to produce products with improved usability. 


\section{METHOD}

\section{Overview and Participants}

The purpose of this study was to grasp the characteristics of transfer of mental models with consideration for a variety of users. Tests to examine transfer of mental models were conducted for older and younger adults separately, and the tasks and experimental devices used in these tests were different. We will describe separately the tests for older adults and younger adults in a later section. Thirty older adults took the Mini-Mental State Examination (MMSE) test, which was a simple test to check cognitive ability before the experiment began. Because the result of the MMSE indicated that one participant might have dementia, the participants were 29 older adults ( 14 men, 15 women, $M_{\text {age }}=68.9, S D=2.6$ ) who would be compensated for the test. Thirty-seven younger adults $\left(20\right.$ men, 17 women, $\left.M_{\text {age }}=21.1, S D=1.7\right)$ participated in this test without compensation.

In this test, we adapted digital cameras as the experimental devices. We chose the digital cameras for several reasons. First, this experiment needed to accommodate a variety of users. In short, we needed to prepare a test that elderly people could attend easily. Although we previously have tried to conduct a test with the computer mouse and PC monitor, it took about 20 minutes for some elderly people to understand the principle of action, and most of them could not move the cursor smoothly. We also have previously examined a test composed of fictional interfaces that do not exist in the real world with a touch screen. Most of the participants likely did not understand the intention of the test because it was difficult for them to image something that was not real. We also previously investigated a test that would duplicate an interface of a digital camera with a touch screen. Some participants did not understand that the interface of digital cameras was shown on the touch-screen monitor; this difficulty was probably related to the participants not having much experience with PCs or other electrical devices. Therefore, we needed to prepare an experimental device that would be easy for the participants to image and operate (i.e., construct mental models). We then adapted real digital cameras because the participants probably would be familiar with the camera itself, although most of them may not have used digital cameras before.

We chose different tasks and digital cameras for older adults and younger adults for two reasons. First, the test for older adults was rather easy for younger adults because almost all younger adults had used digital cameras before. Therefore, we chose different tasks for the younger adults, which were more difficult as compared to those performed by the older adults. Second, because we knew beforehand that some participants were familiar with the digital camera made by Canon, we chose to adapt the digital camera made by Nikon instead.

We conducted a questionnaire regarding experience with digital cameras before the test began. We found that nine out of 29 older adults had used a digital camera before. Seven of the nine older adults use a digital camera a few times a year, and the others use it more often. In contrast, two out of 37 younger adults had never used digital cameras before, but they had experience with PCs and mobile phones. We began the test after confirming that none of the participants had ever used the specific digital cameras used in the test.

\section{Materials and Procedure}

Two groups were prepared in this test. The first group operated product A and product B (A-B group) in turn, and the second one operated product B and product A (B-A group) in turn. We then compared the operational time for product B between the A-B group and the B-A group. When the operational time of B in both groups was compared, only the operational time for product $\mathrm{B}$ was related to the result in the B-A group; we hereafter refer to the B-A group as simply the B group in this case. During the test, participants were required to perform each task with the experimental device three times to construct a mental model of it. Then, we compared the operational time for B in the first trial in both the A-B group (the transfer group) and the B group (the non-transfer group) to examine how mental models were transferred (See Fig. 1). In addition, we evaluated how well participants constructed mental models for the product by referring to part of the cognitive interview proposed by Geiselman and Fisher (1984). The cognitive interview is mainly composed of asking backward and forward questions in terms of operational procedures. Asking backward is likely effective for grasping how strongly the participants construct mental models because this interview has a strong cognitive load. For instance, we asked participants, "Please answer what you should operate before operating X." If participants answered the question correctly, we described them as people who construct mental models for product A. If they could not answer the question, we taught them again how to operate product A. 


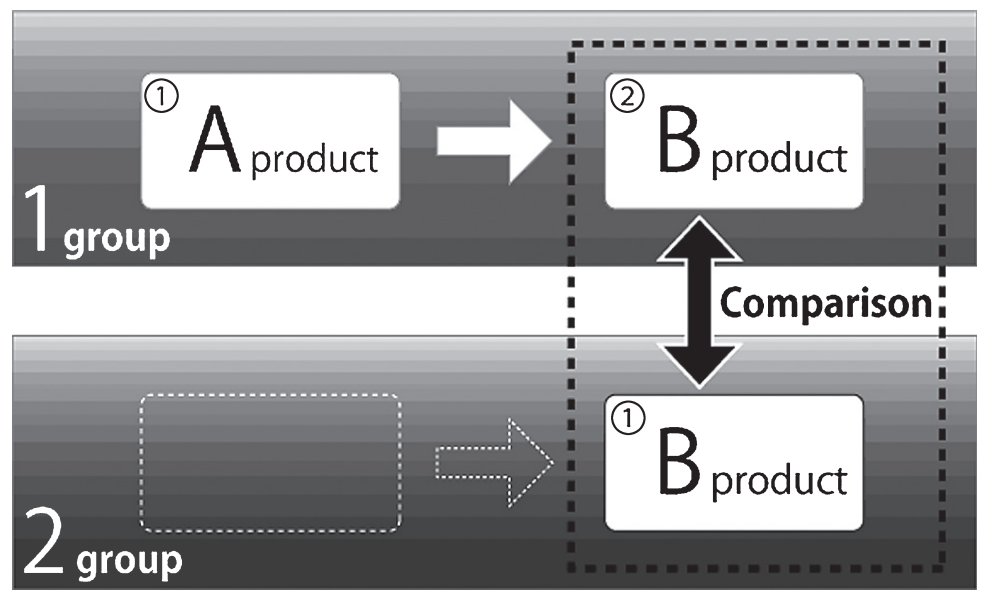

Fig. 1. The experimental approach in this study. First group operates product A and product B in turn. Second group operates product B. Operational time for B in both groups is then compared.

Table 1. Operational Procedures of Experimental Devices for the Older Adults' Test

\begin{tabular}{cll}
\hline Task & \multicolumn{1}{c}{ Panasonic } & \multicolumn{1}{c}{ Canon } \\
\hline 1 & Power-Shooting mode-Shutter & Power-Shooting mode-Shutter \\
2 & Power-Close-up mode-Zoom-Shutter & Power-Shooting mode-Close-up mode-Zoom-Shutter \\
3 & Power-Preview mode-Trash-Delete & Power-Preview mode-Trash-Delete \\
\hline
\end{tabular}

\section{The Test for Older Adults}

In the test for older adults, digital cameras produced by Panasonic (DMC-FX7, hereafter referred to as P) and Canon (IXY DIGITAL IS910, hereafter referred to as C) were adapted. Older adults were divided into two sections: the P-C group (6 men, 8 women) and $\mathrm{C}$ group ( 8 men, 6 women), and the C-P group ( 5 men, 4 women) and $\mathrm{P}$ group (6 men, 9 women). For example, in the $\mathrm{P}-\mathrm{C}$ group, participants first used $\mathrm{P}$ cameras three times and next used $\mathrm{C}$ cameras. In fact, participants of the $\mathrm{P}-\mathrm{C}$ and $\mathrm{P}$ group, and the $\mathrm{C}-\mathrm{P}$ and $\mathrm{C}$ group should be the same because the $\mathrm{P}$ group is supposed to operate $\mathrm{C}$ cameras after operation of $\mathrm{P}$. However, some participants could not achieve the tasks with both experimental devices in the allocated time. Thus, the number of participants is different compared with the $\mathrm{P}-\mathrm{C}$ group and the $\mathrm{P}$ group. This test comprised three tasks:

1. Take a picture with shooting mode.

2. Take a picture with close-up mode and zoom to some extent.

3. Delete the picture that participants took.

Table 1 shows operational procedures of $\mathrm{P}$ and $\mathrm{C}$ to accomplish each task. Operational procedures describe conceptual procedures and do not count detailed steps, such as the number of pushes of a button. To examine characteristics of the transfer of mental models, we compared the operational time for product $\mathrm{C}$ between the $\mathrm{P}-\mathrm{C}$ group and the $\mathrm{C}$ group and compared that of product $\mathrm{C}$ between the $\mathrm{C}$-P group and the $\mathrm{P}$ group.

\section{The test for Younger Adults}

In the test for younger adults, digital cameras of Nikon (COOLPIX P90, hereafter referred to as N) and Panasonic (DMC-FX7, hereafter referred to as P) were adapted. Younger adults were divided into two 
Table 2. Operational Procedures of Experimental Devices for the Younger Adults' Test

\begin{tabular}{cll}
\hline Task & \multicolumn{1}{c}{ Nikon } & \multicolumn{1}{c}{ Panasonic } \\
\hline 1 & Power-Menu-Setup-Sound setup & Power-Menu-Setup-Sound setup \\
2 & Power-Close-up mode-Shutter & Power-Close-up mode-Shutter \\
3 & Power-Scene mode-Menu-Scenery-Shutter & Power-Scene mode-Scenery-Shutter \\
4 & Power-Menu-Preview-Delete & Power-Preview mode-Trash-All Deletion \\
\hline
\end{tabular}

sections: the P-N group (10 men, 8 women) and $\mathrm{N}$ group (10 men, 9 women), and the N-P group (10 men, 9 women) and $\mathrm{P}$ group (10 men, 8 women). This test comprised four tasks:

1. Turn on the sound of operational setup.

2. Take a picture with close-up mode.

3. Take a picture with scenery mode in scene mode.

4. Delete the taken pictures by the function of all image deletion.

Table 2 shows operational procedures of $\mathrm{P}$ and $\mathrm{N}$ to accomplish each task. We compared the operational time for product $\mathrm{N}$ between the $\mathrm{P}-\mathrm{N}$ group and the $\mathrm{N}$ group and that for product $\mathrm{P}$ between the $\mathrm{N}-$ $\mathrm{P}$ group and the $\mathrm{P}$ group.

\section{RESULTS}

To clarify the transfer of mental model, we compared the transfer group and the nontransfer group in terms of operational time. We first conducted a normality test and an F test for operational time of both groups. Then, we performed the Student's t test, Welch's $t$ test, or Mann-Whitney $U$ test for operational time of both groups by considering the result of the normality test and F test. As previous studies (Singley \& Anderson, 1985, 1989) have revealed that operational performance such as deduction of keystroke and planning time could be improved when mental models for a device were transferred well, we defined a task revealing significant differences between groups in operational performance as a task transferring mental models. Figure 2 shows the result of the tests for older adults and younger adults.

\section{The Test for Older Adults}

Figure 2a shows the mean of the operational time for product $\mathrm{C}$ between the $\mathrm{P}-\mathrm{C}$ and $\mathrm{C}$ groups. The Mann-Whitney $\mathrm{U}$ test revealed a significant difference in Task 1, $U=151.5, p=.013$. The Student's t test did not reveal a significant difference in Task 2, $t(26)=-0.93, p=.36$. The difference in Task 3 was not significant with the MannWhitney $\mathrm{U}$ test, $U=57.5, p=.06$. Figure $2 \mathrm{~b}$ shows the mean of the operational time for product $\mathrm{P}$ between the $\mathrm{C}-\mathrm{P}$ and $\mathrm{P}$ groups. The Mann-Whitney $\mathrm{U}$ test revealed a significant difference in Task $1, U=29, p=.02$. The difference in Task 2 was statistically significant with the Welch's t test, $t(22)=-3.47, p=.002$. The Mann-Whitney U test did not reveal a significant difference in Task $3, U=42.5, p=.136$. 
a

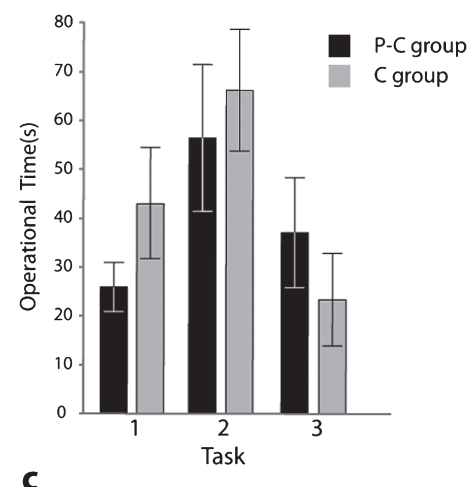

b

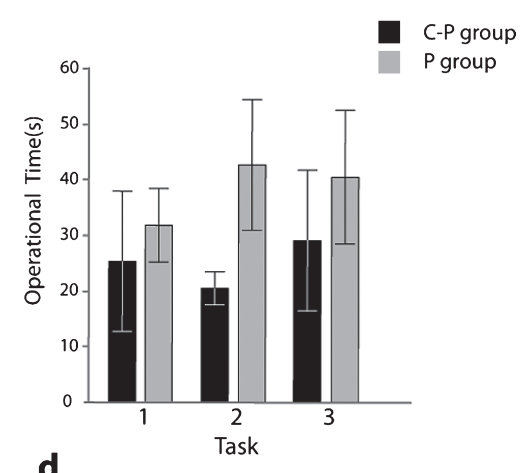

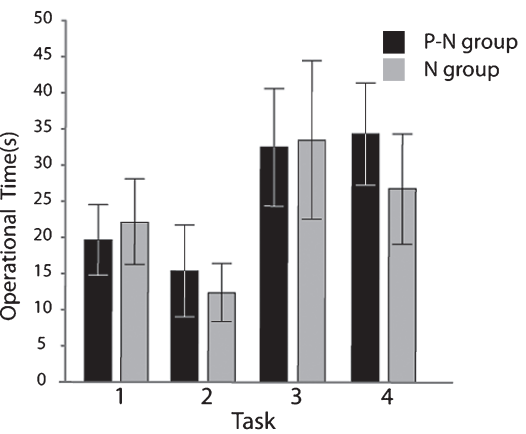

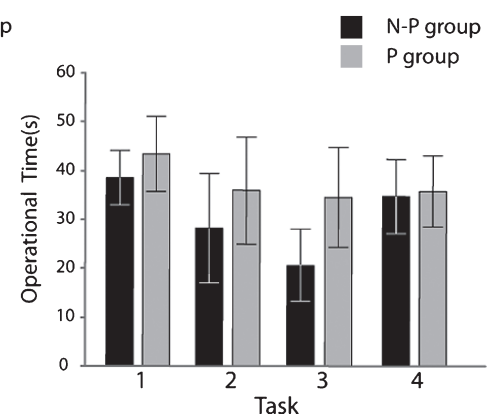

Fig. 2. Comparison of operational time between the transfer group and the non-transfer group. Error bar describes standard deviation of operational time. (a) P-C group and $\mathrm{C}$ group in the test for older adults. (b) C-P group and $\mathrm{P}$ group in the test for older adults. (c) P-N group and $\mathrm{N}$ group in the test for younger adults. (d) N-P group and P group in the test for younger adults.

\section{The Test for Younger Adults}

Figure 2c shows the operational time for product $\mathrm{N}$ between the $\mathrm{P}-\mathrm{N}$ and $\mathrm{N}$ groups. The Mann-Whitney U test did not reveal significant differences for Tasks 1 through 3 , $U=192.5, p=.74 ; U=135, p=.13$; and $U=163, p=.40$, respectively. Student's t test did not reveal significant differences for Task $4, t(35)=1.55, p=.06$. Figure $2 \mathrm{~d}$ shows the operational time of product $\mathrm{P}$ between the N-P and P groups. Student's $\mathrm{t}$ test did not reveal significant differences in Task 1 and Task $4, t(35)=-1.11, p=.13$; and $t(35)=-$ $0.195, p=.42$, respectively. The difference in Task 2 also was not statistically significant by the Mann-Whitney $\mathrm{U}$ test, $U=141.5, p=.18$. The Mann-Whitney $\mathrm{U}$ test revealed significant differences in Task $3, U=85.5, p=.004$.

\section{DISCUSSION}

\section{The Test for Older Adults}

For Task 1 in the $\mathrm{C}-\mathrm{P}$ and $\mathrm{P}$ group, and $\mathrm{P}-\mathrm{C}$ and $\mathrm{C}$ group, the operational time of the 
transfer group (the C-P group and the P-C group) was significantly faster than that of the non-transfer group (the $\mathrm{P}$ group and the $\mathrm{C}$ group). Therefore, the transfer of mental models occurred successfully in these sections. As Table 1 shows, this finding is likely due to learning effect because the operational procedures were the same between $\mathrm{P}$ and $\mathrm{C}$. This result suggests that congruency of operational procedures can bring positive transfer of mental models.

Although there were no significant differences in Task 2 in the $\mathrm{P}-\mathrm{C}$ and $\mathrm{C}$ groups, the difference between the $\mathrm{C}-\mathrm{P}$ and $\mathrm{P}$ groups was statistically significant. This result suggests that constructed mental models for $\mathrm{C}$ can be transferred well in operating $\mathrm{P}$. In this case, the difference in operational procedures between $\mathrm{P}$ and $\mathrm{C}$ needs to be discussed. As Table 1 shows, the difference in Task 2 between $P$ and $C$ is whether "shooting mode" is required (i.e., P [Power-Close-up mode-Zoom-Shutter], C [Power-Shooting mode-Close-up mode-Zoom-Shutter]). Participants can directly choose close-up mode in operating P, but they need to choose shooting mode first in operating C. Therefore, this result suggests that reducing the number of operational procedures could trigger the transfer of mental models in the case that the other operational procedures are congruent with each other.

In Task 3, the mean operational time of the transfer group tended to be inferior to that of the non-transfer group in the $\mathrm{P}-\mathrm{C}$ group and $\mathrm{C}$ group. This finding may suggest that constructed mental models for $\mathrm{P}$ worked as a distraction in operating $\mathrm{C}$. As Table 1 shows, procedures of $\mathrm{P}$ actually correspond to those of $\mathrm{C}$ (i.e., $\mathrm{P}$ [Power-Preview modeTrash-Delete], C [Power-Preview mode-Trash-Delete]). Despite their operational procedures being congruent with each other, we could not find positive transfer or learning effect. To clarify the differences between them, we discuss the flow line in their operation. Figure 3 shows how to accomplish Task 3 in $\mathrm{P}$ and $\mathrm{C}$. As you can see from Figure 3, there are no large differences between them in terms of flow line. Therefore, there likely are other factors distracting the transfer of mental models, such as understanding or finding of an icon. Elderly people may not be good at finding the icon of "Trash" if they are not familiar with icon. For example, we observed some remarks by the transfer group in Task 3, such as "I found the icon of Trash" or "There must be the icon of Trash, but I cannot find it." Judging from these comments, it was likely that they had a mental model that the surface of the digital camera has an icon for trash, and they persisted in finding it or they were not used to looking for what to find. However, we cannot specify the possible reasons for distraction of the transfer in this report. It is necessary to investigate this question further in a future study.

\section{The Test for Younger Adults}

As the results showed, we could not find numerous transfers of mental models. This finding is likely because most of the younger adults had more experience with digital cameras as compared with the older adults. We therefore discuss only Task 3 and Task 4 here.

Although there were no significant differences in Task 3 in the P-N and $\mathrm{N}$ group, the difference in the N-P and $\mathrm{P}$ group was statistically significant. This result suggests that constructed mental models for $\mathrm{N}$ can be transferred well in operating P. As Table 2 shows, 

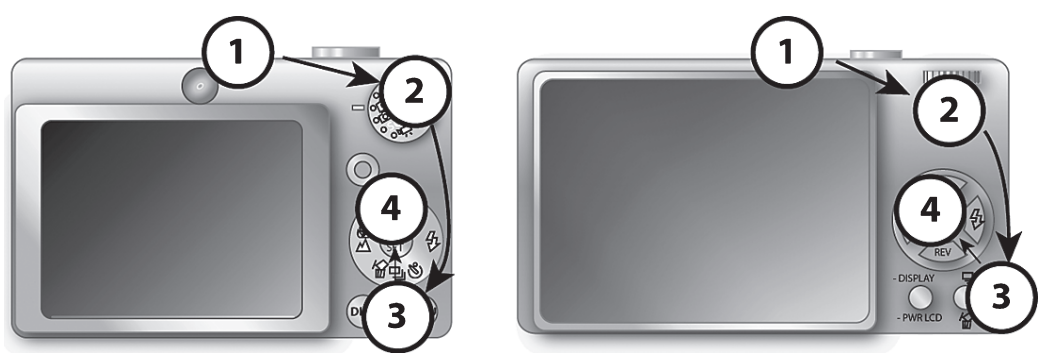

Fig. 3. Operational procedures and route of process of Canon (left) and Panasonic (right). Operational procedures of Canon and Panasonic are as follows "1. Power, 2. Preview mode, 3. Trash, 4. Delete."

the difference in Task 3 between $\mathrm{N}$ and $\mathrm{P}$ is whether the procedures include the use of "Menu" (i.e., N [Power-Scene mode-Menu-Scenery-Shutter], P [Power-Scene modeScenery-Shutter]). Users can directly choose the scenery mode in operating P. Therefore, this result suggests that reducing the number of operational procedures could trigger the transfer of mental models if the other operational procedures are congruent with each other. This common finding between the tests for older adults and younger adults is notable.

In Task 4, we found that operational time of the transfer group (the P-N group) tended to be longer than that of the non-transfer group (the $\mathrm{N}$ group). This result suggests that constructed mental models for $\mathrm{P}$ can be distractions in operating N. Table 2 shows the operational procedures (i.e., $\mathrm{N}$ [Power-Menu-Preview-Delete], C [Power-Preview mode-Trash-All Deletion]); the explanation for the lack of the transfer of mental models is likely the differences in operational procedures. This result suggests that once participants become familiar with a specific procedure, they probably tend to persist in their familiar way, and the transfer of mental models does not occur well. In short, they first try to transfer their familiar mental models in operating another device; they then adjust their mental models after they realize that they cannot accomplish their goal by adapting their familiar mental models. In this case, the findings suggest that negative transfer occurs, so to speak. Negative transfer is defined here as the operational time of the transfer group becoming longer despite having some experiences with similar devices. Negative transfer will occur when constructed mental models work as distractions. Bhavnani and John (2000) pointed out that people tend to persist in using ways that are familiar for them even though they know the most efficient way to achieve a purpose when they operate some complex devices. Moreover, Besnard Greathead, and Baxter (2004) remarked that people tend to wait for consistent information instead of looking for contradictory evidence, which is called confirmation bias. It is said that people overlook contradictory data as confirmation bias. This is one explanation for flawed mental models. In addition, most people are likely to reject any information that is not consistent with their expectations, rather than update their mental models, because people try to avoid the cost of revising their mental models. However, we could not find specific 
properties of the negative transfer of mental models in this study. Because several works regarding wrong mental models have been examined, it is essential to examine the mechanism of negative transfer of mental models in a future study.

\section{The Characteristics of the Transfer of Mental Models}

Taken together, we found that reducing the number of operational procedures can trigger positive transfer of mental models if the other operational procedures are congruent with each other as a characteristic of the transfer of mental models. It is striking that the characteristic of the transfer of mental models is common for both the elderly people and younger adults. In fact, the operational time for younger adults is much better than that for older adults because most younger adults have more experience with digital cameras than older adults do. Therefore, it is reasonable to think that younger adults probably have rich mental models in the specific context of using electronic devices like digital cameras. Some studies (Galotti Baron, \& Sabini, 1986; Staggers \& Norcio, 1993; Nong \& Gavriel, 1996) examining the mental models of experts and novices have revealed that the most notable difference between the two groups is the degree of abstraction. Although most experts have abstract mental models, most novices do not have abstract mental models; they have specific mental models such that they know exactly the operational procedures of experimental tasks. These researchers pointed out that the mental models of experts and novices were different. However, the results of our study suggest that experience does not influence the transfer of mental models. In addition, it seems that the characteristic of positive transfer of mental models is common in older adults and younger adults; that is, the transfer of mental models is not influenced by age. Jonassen and Philip (1996) suggested following components of mental models in addition to the characteristics of mental models proposed by Carley and Palmquist (1992):

1. Structural knowledge

2. Performance/procedural knowledge

3. Reflective procedural knowledge

4. Image of system

5. Metaphors

6. Executive knowledge

Judging from the components of mental models, we believe the characteristic of the transfer of mental models likely corresponds to 2) performance/procedural knowledge.

\section{Conclusion and Future Study}

In conclusion, we found that reducing the number of operational procedures could trigger the positive transfer of mental models if the other operational procedures were congruent with each other. To take advantage of this finding in product development, it will be necessary to reduce the number of operational procedures. However, this change will not be effective if task $\mathrm{X}$ (a-b-c-d-e) is updated to task Y (a-f-j-h). Positive transfer will happen if task $\mathrm{X}$ (a-b-c-d-e) is changed to task $\mathrm{Y}$ (a-c-d-e); that is, if the other operational procedures are congruent with each other.

As Jonassen and Philip (1996) showed, it is likely that some components of mental 
models exist. Similarly, there are probably several components of the transfer of mental models. For example, Zhang and Norman (1994) reported the nature of external representations. They showed that external representation could provide not only memory aids, but also information that can be directly perceived and used without being interpreted and formulated explicitly. Thus, it is reasonable to think that we take advantage of external resources in regard to the unconscious when we transfer our mental models. Although research examining interaction between external resources and mental models has not been developed owing to the difficulty in researching external resources, it is worth investigating in a future study. Hence, we expect to examine not only the transfer of mental models, but also interaction between external resources and mental models in the future.

\section{REFERENCES}

Baltes, P. B. 1987. Theoretical propositions of life-span developmental psychology: On the dynamics between growth and decline. Developmental Psychology, 23, 611-626.

Besnard, D., Greathead, D., \& Baxter, G. 2004. When mental models go wrong: co-occurrences in dynamic, critical systems. International Journal of Human-Computer Studies, 60, 117-128.

Bhavnani, S. K., \& John, B.E. 2000. The strategic use of complex computer systems. Human Computer Interaction, 15, 107-137.

Borgman, C. L. 1986. The user's mental model of an information retrieval system: an experiment on a prototype online catalog. International Journal of Man-Machine Studies, 24, 47-64.

Carley, K., \& Palmquist, M. 1992. Extracting, representing, and analyzing mental models. Social Forces, 70, 601-636.

De Kleer, J., \& Brown, J. 1983. Assumptions and ambiguities in mechanistic mental models. In D. Gentner \& A. L. Stevens (Eds.), Mental models. Lawrence Erlbaum Associates.

Doyle, J.K., \& Ford, D.N. 1998. Mental models concepts for system dynamics research. System Dynamics Review, 14, 3-29.

Forbus, R. 1983. Qualitative reasoning about space and motion. In D. Gentner \& A.L. Stevens (Eds.), Mental models. Lawrence Erlbaum Associates .

Furuta, T., \& Komazaki, H. 1995. Processes of spontaneous formation of mental models and its equivalence to the target (in Japanese). Cognitive Studies, 2, 86-95.

Galotti, K. M., Baron, J., \& Sabini, J. P. 1986. Individual differences in syllogistic reasoning: Deduction rules or mental models? Jornal of Experimental Psychology, 115, 16-25.

Geiselman R. E., \& Fisher, R. P. 1984. Enhancement of eyewitness memory: An empirical evaluation of the cognitive interview. Journal of Police and Science Administration, 12, 74-80.

Gentner, D., \& Gentner, D. R. 1983. Flowing water or teeming crowds: Mental models of electricity. In D. Gentner \& A. L. Stevens (Eds.), Mental models. Lawrence Erlbaum Associates .

Halasz, F. G., \& Moran,T. P. 1983. Mental models and problem solving in using a calculator. Proceedings of the SIGCHI conference on human factors in computing systems, 212-216.

Johnson-Laird, P. (Eds.) 1983. Mental models. Cambridge, MA: Harvard University Press.

Jonassen, D. H., \& Philip, H. 1996. Mental Models: Knowledge in the Head and Knowledge in the World. Proceedings of 1996 international conference on Learning sciences, 433-438.

Kaiho, H. (Eds.) 1988. Kousureba wakariyasui hyougen-ninaru (Japanese title), Fukumura Shuppan Inc.

Kieras, D. E., \& Bovair, S. 1984. The Role of a Mental Model in Learning to Operate a Device. Cognitive Science, 8, 255-273.

Nakatani, L. H., \& Rohrlich, J. A. 1983. Soft machines: A philosophy of user-computer interface design. Proceedings of the SIGCHI conference on Human Factors in Computing Systems, 19-23.

Nong, Y., \& Gavriel, S. 1996. Expert-novice knowledge of computer programming at different levels of 
abstraction. Ergonomics, 39, 461-481.

Norman, D. A. 1983. Some observations on mental models. In D. Gentner \& A. L. Stevens (Eds.), Mental models. Lawrence Erlbaum Associates.

Okawa, I. 1989. A study of relationships between 'Non-normative life events' and intellectual abilities in elderly people (in Japanese). Japanese Journal of Educational Psychology, 37, 100-106.

Singley, M. K., \& Anderson, J. R. 1985. Transfer of text-editing skill. International Journal of ManMachine Studies, 22, 403-423.

Singley, M. K., \& Anderson, J. R. (Eds.) 1989. The Transfer of Cognitive Skill. Cambridge, MA: Harvard University Press.

Staggers, N., \& Norcio, A. F. 1993. Mental models: concepts for human-comuter interaction research. International Journal of Man-machine, 38, 587-605.

Zhang, J., \& Norman, D. A. 1994. Representations in distributed congnitive tasks. Cognitive Science, 18, $87-122$.

Zhang, X., \& Chignell, M. 2001. Assessment of the effects of user characteristics on mental models of information retrieval systems. Journal of the American Society for Information Science and Technology, 52, 445-459.

(Manuscript received October 10, 2010; Revision accepted December 16, 2010) 